\title{
Activation of formate hydrogen-lyase via expression of uptake [NiFe]-hydrogenase in Escherichia coli BL21(DE3)
}

\author{
Byung Hoon Jo and Hyung Joon Cha*
}

\begin{abstract}
Background: Several recent studies have reported successful hydrogen $\left(\mathrm{H}_{2}\right)$ production achieved via recombinant expression of uptake [NiFe]-hydrogenases from Hydrogenovibrio marinus, Rhodobacter sphaeroides, and Escherichia coli (hydrogenase-1) in E. coli BL21(DE3), a strain that lacks $\mathrm{H}_{2}$-evolving activity. However, there are some unclear points that do not support the conclusion that the recombinant hydrogenases are responsible for the in vivo $\mathrm{H}_{2}$ production.

Results: Unlike wild-type BL21(DE3), the recombinant BL21(DE3) strains possessed formate hydrogen-lyase (FHL) activities. Through experiments using $f d h F$ (formate dehydrogenase-H) or hycE (hydrogenase-3) mutants, it was shown that $\mathrm{H}_{2}$ production was almost exclusively dependent on $\mathrm{FHL}$. Upon expression of hydrogenase, extracellular formate concentration was changed even in the mutant strains lacking $\mathrm{FHL}$, indicating that formate metabolism other than FHL was also affected. The two subunits of $H$. marinus uptake [NiFe]-hydrogenase could activate FHL independently of each other, implying the presence of more than two different mechanisms for FHL activation in BL21(DE3). It was also revealed that the signal peptide in the small subunit was essential for activation of FHL via the small subunit.

Conclusions: Herein, we demonstrated that the production of $\mathrm{H}_{2}$ was indeed induced via native FHL activated by the expression of recombinant hydrogenases. The recombinant strains with [NiFe]-hydrogenase appear to be unsuitable for practical in vivo $\mathrm{H}_{2}$ production due to their relatively low $\mathrm{H}_{2}$ yields and productivities. We suggest that an improved $\mathrm{H}_{2}$-producing cell factory could be designed by constructing a well characterized and overproduced synthetic $\mathrm{H}_{2}$ pathway and fully activating the native $\mathrm{FHL}$ in BL21(DE3).
\end{abstract}

Keywords: Recombinant hydrogenase, Escherichia coli BL21(DE3), Biohydrogen, Formate hydrogen lyase, Hydrogenovibrio marinus

\section{Background}

Hydrogen $\left(\mathrm{H}_{2}\right)$ production via biological means has been considered as a potential source of alternative fuel due to clean and truly renewable processes [1]. Hydrogenases are the key enzymes in microbial $\mathrm{H}_{2}$ metabolism that catalyze the reversible reduction of protons with electrons [2]. Certain limitations of native hydrogenase systems for $\mathrm{H}_{2}$ production (i.e., problems related to substrate (electron donor/acceptor) specificity, oxygen $\left(\mathrm{O}_{2}\right)$ sensitivity, catalytic bias to $\mathrm{H}_{2}$ oxidation, electron partitioning, etc.) have been reported in microorganisms [3], and their

*Correspondence: hjcha@postech.ac.kr

Department of Chemical Engineering, Pohang University of Science and Technology, Pohang 790-784, Korea properties appear to be unable to meet current needs. Therefore, expression and engineering of hydrogenases in heterologous hosts is generally accepted as the most influential approach to modification of enzyme qualities and $\mathrm{H}_{2}$ production efficiency for biotechnological applications [3, 4]. Recombinant expression of hydrogenase not only provides the ability to engineer the $\mathrm{H}_{2}$ metabolism of the host for specific purposes but also could facilitate basic studies on the maturation process of the complex metalloenzyme [4].

Escherichia coli has been widely used as a host microbe for protein expression [5]. This bacterium was also adopted for expression of recombinant hydrogenase in several studies, either for study of hydrogenase 
maturation or for improvement of fermentative $\mathrm{H}_{2}$ production by coupling to the native electron transfer system of $E$. coli [6-10]. In particular, the strain BL21(DE3) (or BL21), which is an optimized host for protein overexpression, can neither produce nor consume $\mathrm{H}_{2}$ (no hydrogenase activity) under the general culture conditions where $\mathrm{K}-12$ derivatives do possess the abilities [11-14]. This observation prompted certain researchers to consider this strain as an ideal host for hydrogenase expression and testing for in vivo $\mathrm{H}_{2}$ production [12-14].

According to the composition of bimetallic active sites, hydrogenases are broadly classified into [FeFe]and [NiFe]-hydrogenases from the standpoint of biotechnological importance. E. coli contains four different [NiFe]-hydrogenases, and among those, hydrogenase- 3 is responsible for $\mathrm{H}_{2}$ production during mixed-acid fermentation [15]. This enzyme forms a formate hydrogen-lyase (FHL) complex together with formate dehydrogenase-H, one of the three formate dehydrogenases of E. coli [16].

Recently, certain studies reported that homologous or heterologous expression of the structural (large and small) subunits of uptake [NiFe]-hydrogenases resulted in construction of recombinant BL21(DE3) derivatives that are capable of producing $\mathrm{H}_{2}$ [17-19]. However, some unclear points arise that do not support the conclusion that the expressed hydrogenases are indeed responsible for the in vivo $\mathrm{H}_{2}$ production of the recombinant strains. Among these points, the most critical is that all of the engineered hydrogenases engage in $\mathrm{H}_{2}$ uptake (consumption) and not production in their native hosts [20-22]. In this work, we tackle this problem using simple biochemical and mutant experiments. We suggest that $\mathrm{H}_{2}$ production in such recombinant systems is almost exclusively dependent on the native FHL of E. coli, and thus, careful characterization of the recombinant hydrogenase systems in BL21(DE3) is required, especially for those designed for in vivo $\mathrm{H}_{2}$ production.

\section{Results and discussion}

\section{Activation of FHL activity in recombinant strains}

Several efforts have been put forth to engineer uptake [NiFe]-hydrogenases in BL21(DE3) strain [17-19]. In these studies, $\mathrm{H}_{2}$ production was demonstrated by expressing structural (large and small) subunits of the hydrogenases in the non- $\mathrm{H}_{2}$ producing $E$. coli strain, and the authors concluded that the engineered, non-native hydrogenases could be used as tools to enhance biohydrogen production in E. coli. However, a critical discussion promptly arises related to the fundamental origin of the produced $\mathrm{H}_{2}$ : (1) The engineered hydrogenases are engaged in $\mathrm{H}_{2}$ uptake and not in $\mathrm{H}_{2}$ production in their native hosts, which means that standard redox potentials of their respective electron acceptors (e.g., cytochrome $b$ ) are expected to be much higher than that of $\mathrm{H}_{2}(-420 \mathrm{mV})$ [23]. Additionally, uptake [NiFe]-hydrogenases generally show high catalytic bias to $\mathrm{H}_{2}$ oxidation $[24,25]$. Thus, even if an uptake [NiFe]-hydrogenase is 'wired' to an electron transport system in E. coli, $\mathrm{H}_{2}$ produced via the non-native pathway is not expected to highly accumulate in a closed batch culture system [12], which is in contrast to the results of high $\mathrm{H}_{2}$ accumulation in the previous studies [17-19]. (2) Addition of hypophosphite, an inhibitor of pyruvate formate-lyase, abolished the $\mathrm{H}_{2}$ production in a recombinant strain expressing E. coli HyaBA (hydrogenase-1) [19]. Moreover, addition of formate greatly increased in vivo $\mathrm{H}_{2}$ production. (3) Full maturation of the expressed hydrogenases is questionable because maturation of [NiFe]-hydrogenase further requires highly specific auxiliary proteins [26].

Putting the theoretical and the experimental clues together, we hypothesized that the BL21(DE3) derivatives produce $\mathrm{H}_{2}$ via a native FHL pathway that is activated by the expression of the recombinant hydrogenases. A test for $\mathrm{H}_{2}$ production using formate as a sole electron source showed that the recombinant strains with the heterologous ( $H$. marinus HoxGK and $R$. sphaeroides HupSL) or homologous (E. coli HyaBA) hydrogenase indeed showed FHL activity, whereas the negative control strain with the parental empty vector exhibited negligible FHL activity as expected (Fig. 1). When we measured formate consumption by the strain with $H$. marinus HoxGK, it was found that the cells consumed $1.6 \pm 0.1 \mathrm{mM}$ formate,

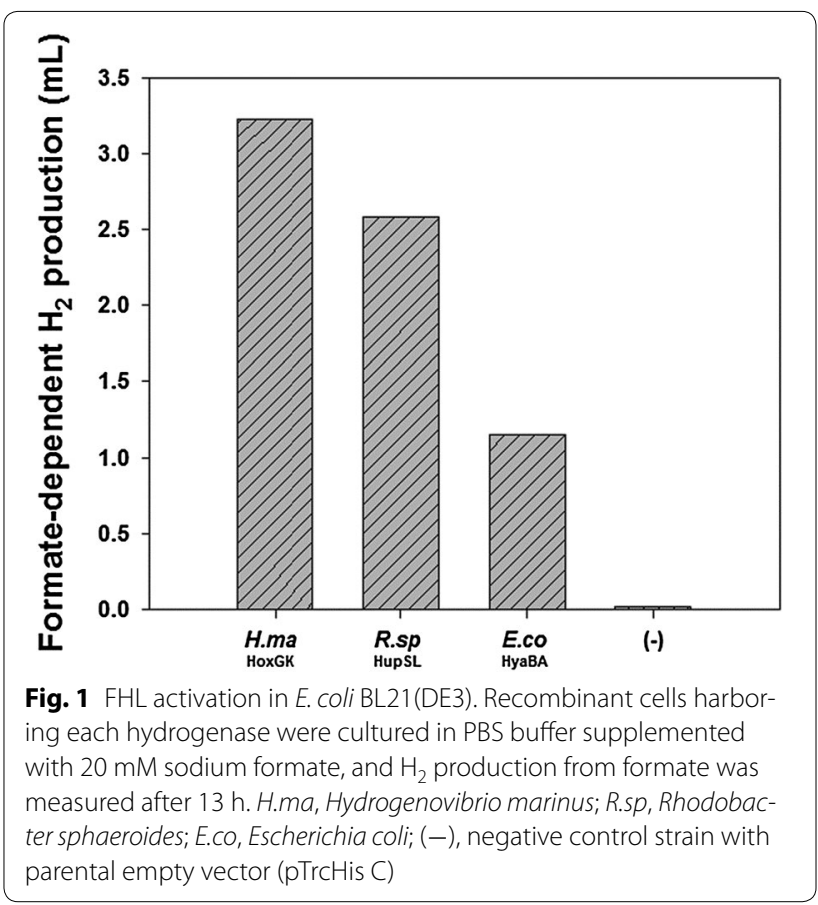


whose corresponding calculated $\mathrm{H}_{2}$ production $(3.58 \mathrm{~mL}$ ) well coincides with the actual amount of $\mathrm{H}_{2}$ production $(3.23 \mathrm{~mL})$. In contrast, the negative control cells showed virtually no consumption of formate $(0.0 \pm 0.1 \mathrm{mM})$. These results imply that the FHL pathway was at least partially responsible for the observed in vivo $\mathrm{H}_{2}$ production in the previously reported recombinant strains.

\section{FHL dependency of $\mathrm{H}_{2}$ production in the recombinant strains}

Measurement of FHL activity was not sufficient to decide whether $\mathrm{H}_{2}$ production in the recombinant strains originates exclusively from the activated FHL pathway. To examine the FHL-dependency, we constructed two knockout BL21(DE3) strains lacking formate dehydrogenase- $\mathrm{H}(f d h F)$ and hydrogenase-3 (hycE), respectively, both of which constitute essential components of the FHL complex [16] and subsequently tested in vivo $\mathrm{H}_{2}$ production by expressing the recombinant hydrogenases.

In the case of the $f d h F$ mutant, all mutant strains produced small amounts of $\mathrm{H}_{2}$ that were roughly comparable to that of the negative control (Fig. 2), which clearly demonstrated that $\mathrm{H}_{2}$ was produced from formate as the only major substrate in the previous reported recombinant strains [17-19]. Similarly, insignificant amounts of $\mathrm{H}_{2}$ were produced by $h y c E$ mutants, which indicates that hydrogenase- 3 was almost entirely responsible for $\mathrm{H}_{2}$ production in the reported BL21(DE3) derivatives (Fig. 2). Although $\mathrm{H}_{2}$ production by both of the mutants with $H$. marinus HoxGK was slightly exceptional (2.1fold for $f d h F$ mutant and 7.5-fold for $h y c E$ mutant compared with the negative controls), the amounts can still

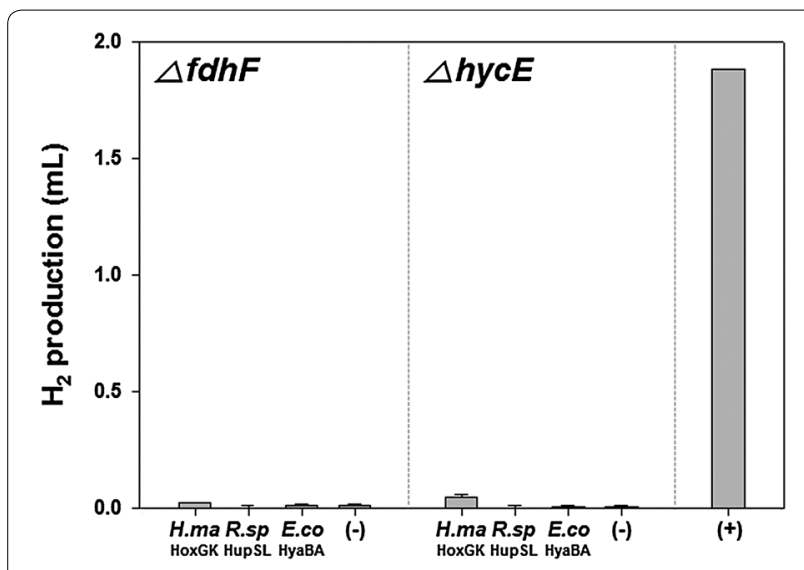

Fig. $2 \mathrm{H}_{2}$ production in FHL-deficient mutant BL21(DE3) strains. Strains lacking formate dehydrogenase-H or hydrogenase-3 were used. H.ma, Hydrogenovibrio marinus; R.sp, Rhodobacter sphaeroides; E.co, Escherichia coli; (-), negative control mutant with parental empty vector (pTrcHis $C) ;(+)$, positive control strain with $R$. sphaeroides HupSL be considered marginal compared with the positive control. It appears that the expression of HoxGK influenced the other $E$. coli hydrogenase system(s) to evolve $\mathrm{H}_{2}$ because $\mathrm{H}_{2}$ was not detected when the E. coli MW1001 strain lacking hydrogenase-1, hydrogenase-2, and hydrogenase-3 was transformed with pTrcHoxGK (data not shown). Thus, we concluded that $\mathrm{H}_{2}$ was produced almost exclusively via the activated FHL pathway in the BL21(DE3) strains with the recombinant hydrogenases. We strongly suspect that the recent report on $\mathrm{H}_{2}$ production in BL21(DE3) by expression of Rhodopseudomonas palustris [NiFe]-hydrogenase [27] falls within this category. It is noteworthy that all recombinant [NiFe]-hydrogenases that activated FHL belong to Group 1 according to the widely used classification of hydrogenases [28].

After in vivo $\mathrm{H}_{2}$ production in the wild-type and the mutant BL21(DE3) strains with H. marinus HoxGK, extracellular formate concentrations were measured and compared with those of negative controls (Table 1). All of the strains with the parent vector showed similar formate level regardless of the FHL mutations. This is not surprising because formate consuming pathways are already impaired in BL21(DE3) [11]. On the other hand, when $H$. marinus HoxGK was expressed, the formate concentration of wild-type BL21(DE3) was lower than those of the mutant strains, indicating that formate was consumed for $\mathrm{H}_{2}$ production. Notably, the overall formate level was lowered upon the expression of hydrogenase even in the mutants that cannot produce $\mathrm{H}_{2}$, which implies that formate metabolism (either production or consumption) other than FHL pathway was also affected by the expression of recombinant hydrogenase.

\section{Involvement of each subunit in FHL activation}

In an effort to reveal the role of uptake [NiFe]-hydrogenase in FHL activation, we investigated the contribution of each subunit to $\mathrm{H}_{2}$ production using $H$. marinus hydrogenase as a model enzyme. Expression vectors were constructed for five different combinations of the large subunit (HoxG), small subunit (HoxK), and small subunit without signal peptide (HoxK*) (Fig. 3a), and all of the subunits with $\mathrm{His}_{6}$-tag were successfully expressed in E. coli BL21(DE3) (Fig. 3b). As shown in Fig. 3c, different amounts of $\mathrm{H}_{2}$ were produced by the different

Table 1 Extracellular concentration of formate $(\mathrm{mM})$ measured after in vivo $\mathrm{H}_{2}$ production in BL21(DE3) derivatives

\begin{tabular}{|c|c|c|c|}
\hline \multirow[t]{2}{*}{ Plasmid } & \multicolumn{3}{|l|}{ Strain } \\
\hline & Wild-type & $\Delta f d h F$ & $\Delta h y c E$ \\
\hline pTrcHis C & $15.9 \pm 0.3$ & $16.5 \pm 0.1$ & $16.1 \pm 0.4$ \\
\hline pTrcHoxGK & $10.6 \pm 0.2$ & $13.3 \pm 1.1$ & $13.1 \pm 1.4$ \\
\hline
\end{tabular}



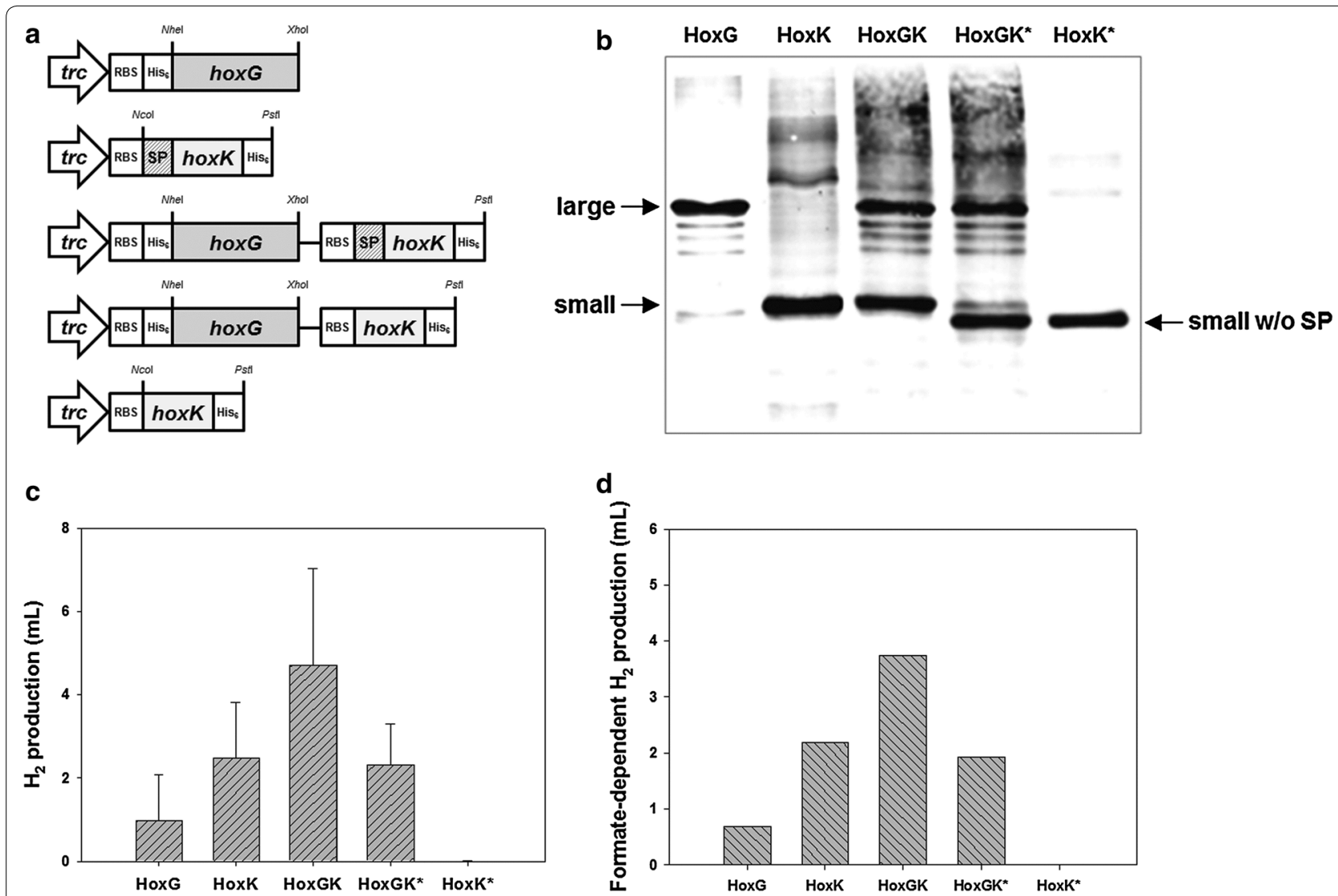

Fig. 3 Combinatorial expressions of hydrogenase subunits of H. marinus in BL21(DE3). a Construction of expression vectors. The constructed vectors (from top to bottom) are pTrcHoxG, pTrcHoxK, pTrcHoxGK, pTrcHoxGK*, and pTrcHoxK*, respectively. b Western blot analysis. Anti-His 6 antibody was used. $\mathbf{c} \mathrm{H}_{2}$ production. d FHL activation. $\mathrm{H}_{2}$ production from formate was measured after 18-h incubation. RBS ribosome binding site, His 6 hexahistidine tag sequence, SP sequence for signal peptide, HoxK* HoxK without signal peptide

combinations. This pattern of $\mathrm{H}_{2}$ production was well correlated with FHL activity $\left(R^{2}>0.99\right)$ (Fig. 3d), implying that the different amounts of $\mathrm{H}_{2}$ production was due to different degrees of FHL activation. Intriguingly, $\mathrm{H}_{2}$ production was observed in the recombinant strains with HoxG or HoxK alone (Fig. 3c). Because the catalytic active site of [NiFe]-hydrogenase is located in large subunit [28], the result of $\mathrm{H}_{2}$ production with only the small subunit corroborates the previous conclusion that the recombinant hydrogenase was not the catalyst that produced $\mathrm{H}_{2}$ in BL21(DE3). Notably, the effects of the two subunits seemed to be additive (Fig. 3c), possibly representing the presence of more than two separate mechanisms for FHL activation. The fact that $\mathrm{H}_{2}$ was produced with HoxG alone also supports this possibility.

The deletion of signal sequence on HoxK resulted in no $\mathrm{H}_{2}$ production, indicating that the signal peptide was essential for FHL activation via the small subunit (Fig. 3c). This observation is consistent with the previous report, in which the importance of signal peptide on in vivo $\mathrm{H}_{2}$ production was shown [17]. Because the signal peptide is implicated in the interaction with membrane component(s) for protein translocation [29], it is likely that the mechanism by which the small subunit activates FHL involves a membrane component that directly or indirectly affects FHL, which is also a membrane protein complex [16].

A recent study on metabolic deficiencies of BL21(DE3) suggested that the lack of FHL activity in BL21(DE3) can be restored by complementation of a wild type copy of fur gene and a high concentration of metal ions $(500 \mu \mathrm{M}$ nickel and $1 \mathrm{mM}$ molybdenum) [11]. In our experiments, no additional ions were added except for $30 \mu \mathrm{M}$ nickel and iron, and little possibility exists that the expressed subunits can function as FNR. Additionally, the effect of FHL restoration by FNR was only partial when compared with the FHL activity of $E$. coli $\mathrm{K}-12$ strains [11]. Intriguingly, an fnr mutant of K-12 strain (PB1000) still possessed $20 \%$ FHL activity of the parent strain [11]. Thus, although we do not offer any clear explanation of how the subunits activate FHL, we suggest the existence of an unknown pathway(s) for FHL activation and regulation 
Table 2 Comparison of $\mathrm{H}_{2}$ production by $E$. coli strains

\begin{tabular}{|c|c|c|c|c|}
\hline Host & Genetic modification & $\begin{array}{l}\mathrm{H}_{2} \text { yield } \\
\left.\text { (mol- } \mathrm{H}_{2} / \text { mol-glucose }\right)\end{array}$ & $\begin{array}{l}\mathrm{H}_{2} \text { productivity } \\
\left(\mathrm{mL}-\mathrm{H}_{2} / \mathrm{L} \text {-culture } \mathrm{h}\right)\end{array}$ & References \\
\hline E. coli BL21(DE3) & H. marinus hoxGK & 0.65 & 25.1 & {$[17]$} \\
\hline E. coli BL21(DE3) & R. sphaeroides hupSL & 0.28 & 19.7 & {$[18]$} \\
\hline E. coli BL21(DE3) & E. colihyaBA & 0.32 & 12.5 & {$[19]$} \\
\hline E. coli BL21(DE3) & R.palustris hupSL & 0.32 & 39.9 & {$[27]$} \\
\hline E. coli BW25113 & $\triangle$ hycA $\triangle$ hyaAB $\triangle$ hybBC $\Delta / d h A \Delta$ frd $A B$ & 1.80 & 420.7 & {$[30,31]$} \\
\hline E. coli BW25113 & $\Delta$ hyaB $\Delta$ hybC $\Delta$ hycA $\Delta$ fdoG $\Delta$ frdC $\Delta / d h A \Delta a c e E$ & 1.32 & 354.8 & {$[32]$} \\
\hline
\end{tabular}

of formate metabolism that is distinct from the $f n r$-mediated activation.

\section{Implications for future research}

The main purpose of engineering hydrogenase or its relevant pathway is to enhance $\mathrm{H}_{2}$ yield and/or productivity. Because $\mathrm{H}_{2}$ production in the recombinant BL21(DE3) strains almost entirely depends on native FHL, in principle, the yield cannot exceed the theoretical maximal $\mathrm{H}_{2}$ yield from formate ( $2 \mathrm{~mol}-\mathrm{H}_{2} /$ mol-glucose $)$ that has been almost realized with $E$. coli K-12 mutant (Table 2). In terms of productivity, the recombinant strains are also much less effective than previously constructed K-12 derivatives (Table 2). Therefore, in their present form, the reported BL21(DE3) strains with the recombinant uptake [NiFe]-hydrogenases appear to be poorly suited for practical in vivo $\mathrm{H}_{2}$ production unless non-native FHL-independent $\mathrm{H}_{2}$ pathways are constructed with the recombinant hydrogenases using synthetic biology and/ or metabolic engineering approaches. Thus, we suggest that recombinant hydrogenase systems designed for in vivo $\mathrm{H}_{2}$ production should be carefully characterized, especially if $E$. coli BL21(DE3) is used as a host; mere observation of in vivo $\mathrm{H}_{2}$ production doesn't imply successful construction of non-native $\mathrm{H}_{2}$ pathway.

E. coli BL21(DE3) is an important strain as a general choice for overexpression of recombinant proteins [5] and holds promise for metabolic engineering and biofuel production. Complete elucidation of the mechanisms for FHL activation in BL21(DE3) is important because it could enable the efficient expansion of $\mathrm{H}_{2}$ yield with high productivity in E. coli; $\mathrm{H}_{2}$ might be produced using more than two substrates simultaneously in BL21(DE3) e.g., via the fully activated FHL pathway and the other FHLindependent $\mathrm{H}_{2}$ pathway that is robustly constituted by recombinant overexpression of $\mathrm{H}_{2}$ metabolizing enzymes [8].

\section{Conclusions}

In this study, the $\mathrm{H}_{2}$ production pathway was investigated in recombinant $E$. coli BL21(DE3) strains that express the structural subunits of uptake [NiFe]-hydrogenase from $H$. marinus (HoxGK), R. sphaeroides (HupSL), or E. coli (HyaBA). The recombinant strains clearly showed FHL activity, whereas the wild-type strain did not. The $\mathrm{H}_{2}$ production was not observed in the recombinant strains lacking $f d h F$ or $h y c E$, thus demonstrating exclusive dependence of the $\mathrm{H}_{2}$ production on activated native FHL. Formate level was changed upon expression of hydrogenase even in the mutant strains lacking FHL, indicating that formate metabolism other than FHL was also affected. Through combinatorial expression of hydrogenase subunits, it was shown that each subunit could activate FHL independently. In addition, it was revealed that the signal peptide is required for FHL activation by the small subunit. The FHL dependence of the recombinant BL21(DE3) derivatives fundamentally limits the practical use of the strains in applications for biohydrogen production. A more effective system might be constructed by synergetic combination of an overproduced synthetic $\mathrm{H}_{2}$ pathway with the fully activated FHL pathway in E. coli BL21(DE3).

\section{Methods}

\section{Strains and plasmid construction}

The strains, plasmids, and primers used in this study are listed in Table 3. All of the DNA works were performed using E. coli TOP10 (Invitrogen, USA), and E. coli BL21(DE3) (Novagen, USA) was used for hydrogenase expression and $\mathrm{H}_{2}$ production. The plasmid for expression of Rhodobacter sphaeroides HupSL (pEMBTL-HJ2) [18] and the E. coli mutant strain MW1001 [33] were kindly provided by Dr. Jiho Min (Chonbuk National University, Jeonju, Korea) and Dr. T. K. Wood (Texas A \& M University, Texas, USA), respectively. The vectors for expression of the hydrogenase subunits of Hydrogenovibrio marinus [34] were constructed by polymerase chain reaction (PCR)-based cloning procedures using genomic DNA of $H$. marinus (DSM 11271) and the listed primers with NheI, NcoI, XhoI, or PstI restriction sites. The PCR products were inserted into the pGEM-T Easy vector (Promega, USA) prior to subcloning into pTrcHis $\mathrm{C}$ 
Table 3 E. coli strains, plasmids, and primers used in this study

\begin{tabular}{|c|c|c|}
\hline Strains, plasmids, or primers & Genotypes, relevant characteristics, or sequences & $\begin{array}{l}\text { Source or } \\
\text { references }\end{array}$ \\
\hline \multicolumn{3}{|l|}{ Strains } \\
\hline TOP10 & $\begin{array}{l}\mathrm{F}^{-} \text {mcrA } \Delta(\text { mrr-hsdRMS-mcrBC) } \Phi 80 \text { lacZ } \Delta M 15 \Delta \text { lacX74 recA1 araD139 } \Delta \text { (ara-leu) } 7697 \text { galU galk } \\
\left.\text { rpsL (Str }{ }^{R}\right) \text { endA1 nupG, streptomycin-resistant }\end{array}$ & Invitrogen \\
\hline BL21(DE3) & F- ompThsdS $S_{B}\left(r_{B}^{-} m_{B}^{-}\right)$gal dcm $\lambda(D E 3)$, carrying the T7 RNA polymerase gene & Novagen \\
\hline $\mathrm{JHO}$ & BL21(DE3) $\Delta$ fdhF::FRT-kan-FRT & This study \\
\hline $\mathrm{JH} 1$ & BL21(DE3) $\Delta h y c E:: F R T-k a n-F R T$ & This study \\
\hline MW1001 & 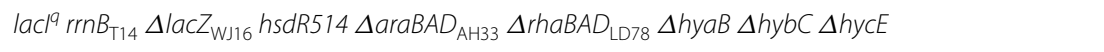 & [33] \\
\hline \multicolumn{3}{|l|}{ Plasmids } \\
\hline pGEM-T Easy & bla lacZ, TA cloning vector & Promega \\
\hline pEMBTL-HJ2 & Expression vector with T7 promoter carrying R. sphaeroides hupS and hupL & [18] \\
\hline pTrc-EcH1ABHis & Expression vector with trc promoter carrying E. coli hyaB and hyaA & [19] \\
\hline pTrcHis C & pBR322 ori bla lac19 , a parental expression vector with trc promoter & Invitrogen \\
\hline pTrcHoxGK & pTrcHis C carrying H. marinus hoxG and hoxK & This study \\
\hline pTrcHoxG & pTrcHis C carrying H. marinus hoxG & This study \\
\hline pTrcHoxk & pTrcHis C carrying H. marinus hoxk & This study \\
\hline pTrcHoxGK* & pTrcHis $\mathrm{C}$ carrying $H$. marinus hoxG and hoxK without signal sequence & This study \\
\hline pTrcHoxk* & pTrcHis C carrying H. marinus hoxK without signal sequence & This study \\
\hline pKD46 & bla $\gamma \beta$ exo araC, Red recombinase vector containing temperature-sensitive replicon & CGSC \\
\hline pKD13 & bla FRT-kan-FRT, template plasmid used for Red recombination & CGSC \\
\hline \multicolumn{3}{|l|}{ Primers $^{\mathrm{a}}$} \\
\hline hoxG & $\begin{array}{l}\text { Forward: GCTAGCATGAGCGTATTAAACACACC (Nhel) } \\
\text { Reverse: CTCGAGTTATCGAACCTTGACGGT }(\text { Xhol) }\end{array}$ & This study \\
\hline hoxk_poly & $\begin{array}{l}\text { Forward: CTCGAGTCTGCCCGTATTGCGCGTAAGGAAATCCATTATGTCAT CTCAAGTTGAAAC (Xhol) } \\
\text { Reverse: CTGCAGTCAATGGTGATGGTGATGATGACCGCCTTTATCTCCTT TCTTTTGAGCC (Pstl) }\end{array}$ & This study \\
\hline hoxk__poly & $\begin{array}{l}\text { Forward: CTCGAGTCTGCCCGTATTGCGCGTAAGGAAATCCATTATGGCG AACAAAATTGCTCATGC } \\
\text { GAT (Xhol) } \\
\text { Reverse: ditto }\end{array}$ & This study \\
\hline hoxk & $\begin{array}{l}\text { Forward: CCATGGGCTCATCTCAAGTTGAAACGTT }(\text { Ncol) } \\
\text { Reverse: ditto }\end{array}$ & This study \\
\hline hoxk* & $\begin{array}{l}\text { Forward: CCATGGGCAACAAAATTGCTCATGCGAT (NCOl) } \\
\text { Reverse: ditto }\end{array}$ & This study \\
\hline fdhF13 & $\begin{array}{l}\text { Forward: CAATCACGTACTGCTCGGCGGCGCGCTGATCGGCGATCGGCTCG ACGCGCATTCCGGGG } \\
\text { ATCCGTCGACC } \\
\text { Reverse:TCCTGACCCCGCGCCTGAAAACCCCCATGATCCGTCGCCAGCGT GGCGGCTGTAGGCT } \\
\text { GGAGCTGCTTCG }\end{array}$ & This study \\
\hline hycE13 & $\begin{array}{l}\text { Forward: TTTTTGATAAAGGTAAACATGGCGATTCCTTATTTCAGCGGCGA GTTTTTATTCCGGGG } \\
\text { ATCCGTCGACC } \\
\text { Reverse: TTAGCGTTCGTCTCCTTGCTGGCGGCGTGATTAAAGAGAGTTTG AGCATGTGTAGGCTGG } \\
\text { AGCTGCTTCG }\end{array}$ & This study \\
\hline fdhFchk & $\begin{array}{l}\text { Forward: GTAGGGAGTAACCAGTATAA } \\
\text { Reverse: AATGACCCCACATAAAATGT }\end{array}$ & This study \\
\hline hycEchk & $\begin{array}{l}\text { Forward: CCAGCGGATAAGACGAGGT } \\
\text { Reverse: CGTCTTGATATTACTCCGCG }\end{array}$ & This study \\
\hline
\end{tabular}

a Regions that hybridize to the corresponding template sequences are bolded, and restriction sites are underlined

(Invitrogen). For polycistronic expression of both hydrogenase subunits, the primers hoxK_poly and hoxK"_poly were designed to contain an intergenic sequence with a ribosome binding site (RBS), a slightly modified portion of the intergenic sequence between $l a c Z$ and $l a c Y$ found in the $E$. coli genome. The plasmid pTrcHoxGK was primarily used throughout the study for expression of $H$. marinus hydrogenase. E. coli cells were grown and maintained in Luria-Bertani (LB) medium (Usb Corp., USA) supplemented with the appropriate antibiotics (ampicillin, $50 \mu \mathrm{g} / \mathrm{mL}$; streptomycin or kanamycin, $10 \mu \mathrm{g} / \mathrm{mL}$ ) at $37^{\circ} \mathrm{C}$ in a shaking incubator at $220 \mathrm{rpm}$ (Jeiotech, Korea). 


\section{Construction of mutant strains}

The Red recombination system with pKD46 (Coli Genetic Stock Center (CGSC), USA) was adopted for inactivation of chromosomal $f d h F$ or $h y c E$ gene in E. coli BL21(DE3). A gene construct composed of kanamycin resistance gene $(k a n)$ flanked by FLP recognition target (FRT) sites on pKD13 (CGSC) was amplified by PCR using $f d h F$ - or $h y c E$-specific primers with 50-nt homology extensions. Gene disruption was performed as described in [35] and confirmed by PCR using specific primers that were designed based on the sequences flanking the disrupted region of the genome. The kan gene was not cured to avoid contamination in cell culture.

\section{In vivo $\mathrm{H}_{2}$ production}

The recombinant $E$. coli BL21(DE3) derivatives transformed with the expression vectors were cultured in $100 \mathrm{~mL}$ of $\mathrm{M} 9$ media $\left(6 \mathrm{~g} / \mathrm{L} \mathrm{Na}_{2} \mathrm{HPO}_{4}, 3 \mathrm{~g} / \mathrm{L} \mathrm{K \textrm {K } _ { 2 }} \mathrm{PO}_{4}\right.$, $1 \mathrm{~g} / \mathrm{L} \mathrm{NH}_{4} \mathrm{Cl}, 0.5 \mathrm{~g} / \mathrm{L} \mathrm{NaCl}, 2 \mathrm{mM} \mathrm{MgSO}_{4}$, and $100 \mu \mathrm{M}$ $\mathrm{CaCl}_{2}$ ) supplemented with $5 \mathrm{~g} / \mathrm{L}$ of casamino acids (BD Bioscience, USA), $5 \mathrm{~g} / \mathrm{L}$ of glucose, and $50 \mu \mathrm{g} / \mathrm{mL}$ of ampicillin (and $10 \mu \mathrm{g} / \mathrm{mL}$ of kanamycin only for mutant strains) in $165-\mathrm{mL}$ serum bottles (Wheaton, USA) at $37{ }^{\circ} \mathrm{C}$ and $220 \mathrm{rpm}$. When the cell density reached $\sim 0.6$ $\mathrm{OD}$ at $600 \mathrm{~nm}$, the cultures were induced for hydrogenase expression and $\mathrm{H}_{2}$ production with the addition of $1 \mathrm{mM}$ isopropyl- $\beta$-D-thiogalactopyranoside (IPTG; Carbosynth, $\mathrm{UK}), 30 \mu \mathrm{M} \mathrm{NiSO}$, and $30 \mu \mathrm{M} \mathrm{FeSO}_{4}$. The bottles were tightly sealed with rubber stoppers and aluminum caps and cultivated for a further $16 \mathrm{~h}$ until $\mathrm{H}_{2}$ production was measured using gas chromatography (GC; Younglin Instrument, Korea).

\section{FHL activity assay}

After in vivo $\mathrm{H}_{2}$ production, cells were harvested by centrifugation at $4{ }^{\circ} \mathrm{C}$ and $4000 \times g$ for $10 \mathrm{~min}$ and washed with phosphate buffered saline (PBS; $8 \mathrm{~g} / \mathrm{L} \mathrm{NaCl}, 1.44 \mathrm{~g} / \mathrm{L}$ $\mathrm{Na}_{2} \mathrm{HPO}_{4}, 0.2 \mathrm{~g} / \mathrm{L} \mathrm{KCl}$, and $0.24 \mathrm{~g} / \mathrm{L} \mathrm{KH}_{2} \mathrm{PO}_{4} ; \mathrm{pH} 7.4$ ). They were resuspended in $98 \mathrm{~mL}$ of PBS in the serum bottle with addition of $2 \mathrm{~mL}$ of $1 \mathrm{M}$ sodium formate. Immediately after brief $(\sim 3 \mathrm{~min})$ flushing with $\mathrm{N}_{2}$ gas, the bottle was sealed with a rubber stopper and an aluminum cap. After incubation at $37{ }^{\circ} \mathrm{C}$ and $220 \mathrm{rpm}$, the production of $\mathrm{H}_{2}$ from formate was analyzed from the gas phase of the bottle via GC.

\section{$\mathrm{H}_{2}$ production measurement}

The $\mathrm{H}_{2}$ production was measured as previously described [36]. In brief, a specific volume (usually $100 \mu \mathrm{L}$ ) of gas was sampled from the headspace of culture bottle and analyzed by $\mathrm{GC}$ to determine the partial $\mathrm{H}_{2}$ pressure. The total amount of $\mathrm{H}_{2}$ was calculated by multiplying the
$\mathrm{H}_{2}$ concentration by the headspace volume of the bottle $(65 \mathrm{~mL})$.

\section{Western blot analysis}

Western blot analysis was performed for detection of hexahistidine $\left(\mathrm{His}_{6}\right.$ )-tagged proteins as previously described [36].

\section{Formate measurement}

Formate was measured by enzymatic assay using formate dehydrogenase as previously described [37] with slight modifications. Samples were diluted $1 / 10$ with deionized water. A reaction solution containing $610 \mu \mathrm{L}$ of $80 \mathrm{mM}$ sodium phosphate buffer ( $\mathrm{pH} 7.0$ ), $300 \mu \mathrm{L}$ of $10 \mathrm{mM}$ nicotinamide adenine dinucleotide $\left(\mathrm{NAD}^{+}\right.$; Sigma-Aldrich, USA) and $100 \mu \mathrm{L}$ of formate dehydrogenase $(\sim 1 \mathrm{mg} /$ $\mathrm{mL}$; Sigma-Aldrich) was mixed with $25 \mu \mathrm{L}$ of the diluted sample solution. After $2.5 \mathrm{~h}$ reaction at $37^{\circ} \mathrm{C}$, the absorbance change by formate-dependent $\mathrm{NAD}^{+}$reduction was measured at $340 \mathrm{~nm}$. Formate concentration was calculated based on the absorbance change and a standard curve prepared using sodium formate solutions (SigmaAldrich) with various concentrations.

\section{Authors' contributions}

$\mathrm{BHJ}$ and $\mathrm{HJC}$ designed the research. $\mathrm{BHJ}$ performed the experiments and analyzed the data. $\mathrm{BHJ}$ and $\mathrm{HJC}$ wrote the paper. Both authors read and approved the final manuscript.

\section{Acknowledgements}

This work was supported by the Energy Efficiency and Resources Core Technology Program of the Korea Institute of Energy Technology Evaluation and Planning (KETEP) grant funded by the Korea government Ministry of Trade, Industry and Energy (20142020200980).

\section{Compliance with ethical guidelines}

\section{Competing interests}

The authors declare that they have no competing interests.

Received: 12 July 2015 Accepted: 16 September 2015

Published online: 22 September 2015

\section{References}

1. Lee HS, Vermaas WFJ, Rittmann BE. Biological hydrogen production: prospects and challenges. Trends Biotechnol. 2010;28:262-71.

2. Mertens R, Liese A. Biotechnological applications of hydrogenases. Curr Opin Biotechnol. 2004;15:343-8.

3. Rousset $M$, Liebgott $P$. Engineering hydrogenases for $\mathrm{H}_{2}$ production: bolts and goals. In: Zannoni D, Philippis RD, editors. Microbial BioEnergy: hydrogen production. Dordrecht Netherlands: Springer; 2014. p. 43-77.

4. English CM, Eckert C, Brown K, Seibert M, King PW. Recombinant and in vitro expression systems for hydrogenases: new frontiers in basic and applied studies for biological and synthetic $\mathrm{H}_{2}$ production. Dalton Trans. 2009;45:9970-8.

5. Terpe K. Overview of bacterial expression systems for heterologous protein production: from molecular and biochemical fundamentals to commercial systems. Appl Microbiol Biotechnol. 2006;72:211-22. 
6. Wells MA, Mercer J, Mott RA, Pereira-Medrano AG, Burja AM, Radianingtyas $\mathrm{H}$, et al. Engineering a non-native hydrogen production pathway into Escherichia coli via a cyanobacterial [NiFe] hydrogenase. Metab Eng. 2011;13:445-53.

7. Sun JS, Hopkins RC, Jenney FE, McTernan PM, Adams MWW. Heterologous expression and maturation of an NADP-dependent [NiFe]-hydrogenase: a key enzyme in biofuel production. PLoS One. 2010;5:e10526.

8. Ghosh D, Bisaillon A, Hallenbeck PC. Increasing the metabolic capacity of Escherichia coli for hydrogen production through heterologous expression of the Ralstonia eutropha SH operon. Biotechnol Biofuels. 2013;6:122.

9. Akhtar MK, Jones PR. Construction of a synthetic YdbK-dependent pyruvate: $\mathrm{H}_{2}$ pathway in Escherichia coli BL21(DE3). Metab Eng. 2009;11:139-47.

10. Kim YM, Cho HS, Jung GY, Park JM. Engineering the pentose phosphate pathway to improve hydrogen yield in recombinant Escherichia coli. Biotechnol Bioeng. 2011;108:2941-6.

11. Pinske C, Bonn M, Kruger S, Lindenstrauss U, Sawers RG. Metabolic deficiences revealed in the biotechnologically important model bacterium Escherichia coli BL21(DE3). PLoS One. 2011;6:e22830.

12. Veit A, Akhtar MK, Mizutani T, Jones PR. Constructing and testing the thermodynamic limits of synthetic $\mathrm{NAD}(\mathrm{P}) \mathrm{H}: \mathrm{H}_{2}$ pathways. Microb Biotechnol. 2008;1:382-94.

13. Mishra J, Khurana S, Kumar N, Ghosh AK, Das D. Molecular cloning, characterization, and overexpression of a novel [Fe]-hydrogenase from a high rate of hydrogen producing Enterobacter cloacae IIT-BT 08. Biochem Biophys Res Commun. 2004;324:679-85.

14. Akhtar MK, Jones PR. Deletion of iscR stimulates recombinant clostridial Fe-Fe hydrogenase activity and $\mathrm{H}_{2}$-accumulation in Escherichia coli BL21(DE3). Appl Microbiol Biotechnol. 2008;78:853-62.

15. Redood MD, Mikheenko IP, Sargent F, Macaskie LE. Dissecting the roles of Escherichia coli hydrogenases in biohydrogen production. FEMS Microbiol Lett. 2008;278:48-55.

16. Sawers RG. Formate and its role in hydrogen production in Escherichia coli. Biochem Soc Trans. 2005;33:42-6.

17. Kim JYH, Jo BH, Cha HJ. Production of biohydrogen by heterologous expression of oxygen-tolerant Hydrogenovibrio marinus [NiFe]-hydrogenase in Escherichia coli. J Biotechnol. 2011;155:312-9.

18. Lee SY, Lee HJ, Park JM, Lee JH, Park JS, Shin HS, et al. Bacterial hydrogen production in recombinant Escherichia coli harboring a HupSL hydrogenase isolated from Rhodobacter sphaeroides under anaerobic dark culture. Int J Hydrogen Energy. 2010;35:1112-6.

19. Kim JYH, Jo BH, Cha HJ. Production of biohydrogen by recombinant expression of [NiFe]-hydrogenase 1 in Escherichia coli. Microb Cell Fact. 2010;9:54.

20. Nishihara H, Miyata Y, Miyashita Y, Bernhard M, Pohlmann A, Friedrich B, et al. Analysis of the molecular species of hydrogenase in the cells of an obligately chemolithoautotrophic, marine hydrogen-oxidizing bacterium, Hydrogenovibrio marinus. Biosci Biotechnol Biochem. 2001;65:2780-4.
21. Lukey MJ, Parkin A, Roessler MM, Murphy BJ, Harmer J, Palmer T, et al. How Escherichia coli is equipped to oxidize hydrogen under different redox conditions. J Biol Chem. 2010;285:3928-38.

22. Koku H, Eroglu I, Gunduz U, Yucel M, Turker L. Aspects of the metabolism of hydrogen production by Rhodobacter sphaeroides. Int J Hydrogen Energy. 2002;27:1315-29.

23. Pandelia ME, Lubitz W, Nitschke W. Evolution and diversification of Group 1 [NiFe] hydrogenases. Is there a phylogenetic marker for $\mathrm{O}_{2}$-tolerance? BBA-Bioenergetics. 2012;1817:1565-75.

24. Abou Hamdan A, Dementin S, Liebgott PP, Gutierrez-Sanz O, Richaud P, De Lacey AL, et al. Understanding and tuning the catalytic bias of hydrogenase. J Am Chem Soc. 2012;134:8368-71.

25. Murphy BJ, Sargent F, Armstrong FA. Transforming an oxygen-tolerant [NiFe] uptake hydrogenase into a proficient, reversible hydrogen producer. Energy Environ Sci. 2014;7:1426-33.

26. Casalot L, Rousset M. Maturation of the [NiFe] hydrogenases. Trends Microbiol. 2001;9:228-37.

27. Zhou P, Wang YM, Gao R, Tong J, Yang ZY. Transferring [NiFe] hydrogenase gene from Rhodopeseudomonas palustris into E. coli BL21(DE3) for improving hydrogen production. Int J Hydrogen Energy. 2015;40:4329-36.

28. Vignais PM, Billoud B. Occurrence, classification, and biological function of hydrogenases: an overview. Chem Rev. 2007;107:4206-72.

29. Mergulhao FJM, Summers DK, Monteiro GA. Recombinant protein secretion in Escherichia coli. Biotechnol Adv. 2005;23:177-202.

30. Maeda T, Sanchez-Torres V, Wood TK. Hydrogen production by recombinant Escherichia coli strains. Microb Biotechnol. 2012;5:214-25.

31. Kim S, Seol E, Oh YK, Wang GY, Park S. Hydrogen production and metabolic flux analysis of metabolically engineered Escherichia coli strains. Int J Hydrogen Energy. 2009;34:7417-27.

32. Maeda T, Sanchez-Torres V, Wood TK. Enhanced hydrogen production from glucose by metabolically engineered Escherichia coli. Appl Microbiol Biotechnol. 2007;77:879-90.

33. Maeda T, Sanchez-Torres V, Wood TK. Escherichia coli hydrogenase 3 is a reversible enzyme possessing hydrogen uptake and synthesis activities. Appl Microbiol Biotechnol. 2007;76:1035-42.

34. Jo BH, Hwang BH, Cha HJ. Draft genome sequence of Hydrogenovibrio marinus $\mathrm{MH}-110$, a model organism for aerobic $\mathrm{H}_{2}$ metabolism. J Biotechnol. 2014;185:37-8.

35. Datsenko KA, Wanner BL. One-step inactivation of chromosomal genes in Escherichia coli K-12 using PCR products. Proc Natl Acad Sci USA. 2000;97:6640-5.

36. Jo BH, Kim JYH, Seo JH, Cha HJ. Oxygen-dependent enhancement of hydrogen production by engineering bacterial hemoglobin in Escherichia coli. Int J Hydrogen Energy. 2014;39:10426-33.

37. Triebig G, Schaller KH. A simple and reliable enzymatic assay for the determination of formic acid in urine. Clin Chim Acta. 1980;108:355-60.

\section{Submit your next manuscript to BioMed Central and take full advantage of:}

- Convenient online submission

- Thorough peer review

- No space constraints or color figure charges

- Immediate publication on acceptance

- Inclusion in PubMed, CAS, Scopus and Google Scholar

- Research which is freely available for redistribution

Submit your manuscript at

www.biomedcentral.com/submit
C Biomed Central 